\title{
Tuning of the Extended Concentric Tube Resonators
}

\author{
P. Choudary Chaitanaya and M. L. Munjal \\ Facility for Research in Technical Acoustics (FRITA), Department of Mechanical Engineering, Indian Institute of \\ Science, Bangalore - 560 012, India.
}

(Received 15 June 2010, accepted 24 January 2011)

It has been shown recently that the acoustic performance of the extended tube expansion chambers can be improved substantially by making the extended inlet and outlet equal to half and quarter chamber lengths, duly incorporating the end corrections due to the evanescent higher order modes that would be generated at the discontinuities. Such chambers, however, suffer from the disadvantages of high back pressure and generation of aerodynamic noise at the area discontinuities. These two disadvantages can be overcome by means of a perforated bridge between the extended inlet and extended outlet. This paper deals with design or tuning of these extended concentric tube resonators.

One dimensional control volume approach is used to analyze this muffler configuration. It is validated experimentally making use of the two source-location method. It is thus shown that the inertance of holes plays a role similar to the lumped inertance generated by evanescent 3-D modes at the terminations of the quarter wave resonators in the case of the double-tuned extended tube chambers. The effect of mean flow is also investigated. The resultant transfer matrix is then used to carry out a systematic parametric study in order to arrive at empirical expressions for the correction lengths. Thus, an extended concentric tube resonator can be tuned such that the first three troughs, which characterize the corresponding simple chamber transmission loss (TL) curve, may be eliminated making use of the proposed procedure. In fact, the entire TL curve at low and medium frequencies may be substantially lifted, making the tuned extended concentric tube resonator a viable design option.

\section{INTRODUCTION}

The transmission loss (TL) curve of a highly perforated concentric tube resonator (CTR) is known to be similar to that of a corresponding simple expansion chamber (SEC). The CTR however does not suffer from the disadvantages of high pressure drop and the aerodynamic noise generation that characterize the flow-acoustic performance of an SEC. ${ }^{1}$ It is possible to design double-tuned extended-tube expansion chambers where the quarter wave resonances of the extended inlet and outlet would tune out the TL troughs of the SEC. ${ }^{2,3}$ The present paper seeks to extend the tuning to CTRs.

The concept of end corrections was given by Karal. ${ }^{4}$ The acoustic inertance due to discontinuity can be considered as a correction term to be added to the analogous acoustical inertance of the tube and can be interpreted physically as an increase in the equivalent length of the tube. Sahasrabudhe et al. made use of a finite element analysis to derive general expressions for inertance due to the evanescent modes generated at sudden area discontinuities. ${ }^{5}$ Torregrosa et al. explored the possibility of obtaining a relatively accurate prediction of the acoustic performance of extended-duct and perforatedduct mufflers by introducing suitable end correction into simple one-dimensional models with the help of finite element analysis. They introduced a general correlation in terms of a non-dimensional parameter for multidimensional effects associated with the evanescent higher order modes at a sudden area change. However, for perforated mufflers, a general correlation could not be obtained due to the eventual coupling between the quarter-wave effect and tube-cavity resonance. ${ }^{6}$ Peat discussed the effect of porosity and mean flow on these end corrections for bare perforated pipes with experimental vali- dations with reference to a Helmholtz resonator or theory of simple expansion chamber. ${ }^{7}$ These end corrections are validated for the Helmholtz resonator but cannot be precise for the concentric tube resonator due to several variables and factors that affect the end corrections. An empirical expression for these end corrections, which should be applied at the junction between a plain and a perforated pipe in the absence of mean flow, was given subsequently by $\mathrm{Peat}^{8}$ with respect to the Helmholtz resonator and also validated experimentally. Munjal and Gowri used a 3-D model of analysis (FEM analysis) in order to obtain information on these end corrections and applied the same to the concentric tube resonators for stationary medium. ${ }^{3}$

An inappropriate value of perforate impedance adopted in the 3-D FEM analysis would lead to erroneous end corrections. The expression given by Lee and Ih fills up this lacuna for CTRs. ${ }^{9}$ As a 3-D model has its own limitations, experimental validation is required. The end corrections calculated by Peat are not validated for an extended concentric tube resonator. Munjal and Gowri's 3-D analysis also needs to be validated with measurements.

In this paper, therefore, tuned concentric tube chambers are designed and validated experimentally for an incompressible mean flow, making use of a specially designed setup based on the two source-location method..$^{10,11}$ Of course, one could make use of 3-D analytical approaches for the same purpose. ${ }^{12,13}$ However, an experimental technique has been adopted here. A generalized design procedure is provided in order to tune the extended concentric tube resonators for better attenuation. In particular, values of the correction lengths and end corrections are compared with those available in the liter- 